\title{
Atypical traumatic of pulmonary artery after Rib fractures
}

\section{Massine EL Hammoumi ${ }^{1}$ and E1 Hassane Kabiri ${ }^{2 *}$}

${ }^{1}$ Department of Thoracic Surgery Mohamed V Military University Hospital, Morocco

${ }^{2}$ Faculty of Medicine and Pharmacy, Mohamed V University, Rabat, Morocco

\section{Case report}

A 42-year-old patient without notable history, the victim of a highway accident (pedestrian struck by a truck). Admitted to the emergency for severe chest trauma; on admission, the patient was conscious, polypneic, with abdominal breathing, hemodynamically stable. After setting condition, a chest radiograph performed showed left rib fractures with a dissociated fragment from the chest wall, a left fractured scapula and soft parenchymal opacities (Figure 1).

The Chest CT objectified multiple storied rib fractures of 2nd to 5th left costal arch with a bone fragment in the endothoracic position threatening pulmonary artery, a moderate left pleural effusion, a basement filling with left lung collapse (Figure 2 and 3). A left thoracic drainage extracted $500 \mathrm{cc}$ of blood. The fracture of the scapula was managed by orthopedic treatment. After three days of monitoring in surgical reanimation, the patient respiratory evolution was severe with desaturation to $73 \%$. The arterial gazometry showed a $\mathrm{pH}$ to 7.43 , a $\mathrm{Pa}$ $\mathrm{O} 2$ to $34 \mathrm{mmHg}$ and $\mathrm{PaCO} 2$ to $39,4 \mathrm{mmhg}$. wich posed the indication of intubation with assisted ventilation to normalize gasometry results. Furthermore the patient showed signs of pulmonary infection $\left(\mathrm{T}^{\circ}: 38.2\right.$, purulent secretions, WBC: $16400 \mathrm{e} / \mathrm{mm}^{3}$, CRP: $\left.237 \mathrm{mg} / \mathrm{l}\right)$. Protected distal samples were made and the patient received an antibiotherapy (Amikacin $(1 \mathrm{~g} / 24 \mathrm{~h})+$ Ceftazidime $(1 \mathrm{~g} / 6 \mathrm{H}))$ according to the results of the antibiogram with good clinical and biological evolution. The patient had also respiratory physical therapy with daily tracheal aspirations. At the 5th day the patient was extubated and the chest tube removed.

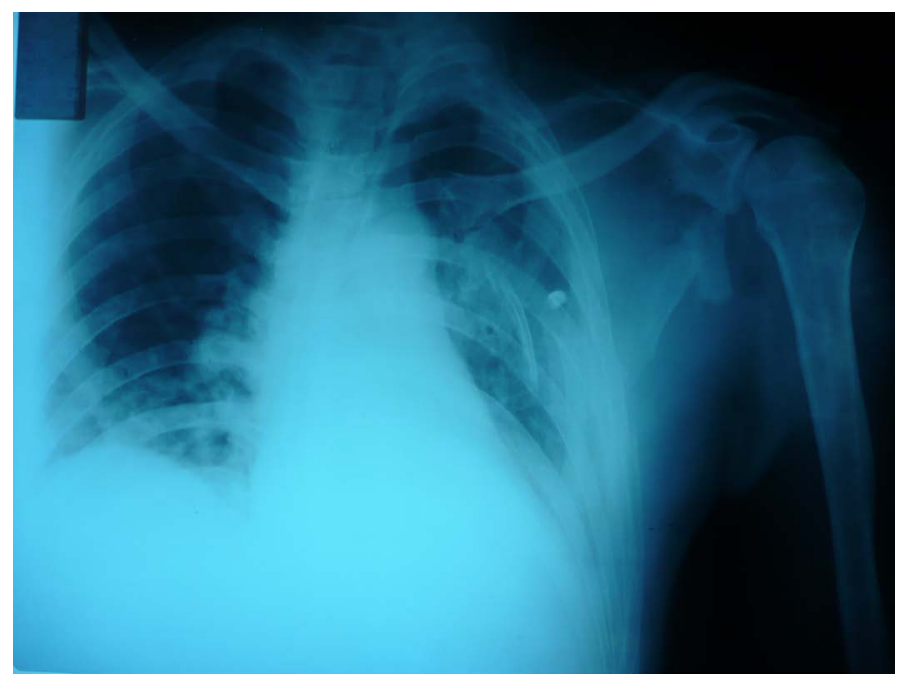

Figure 1. Pulmonary x-ray. Multiple left fracture fractures with bone fragments separated from the thoracic wall and fracture of the homolateral scapula.

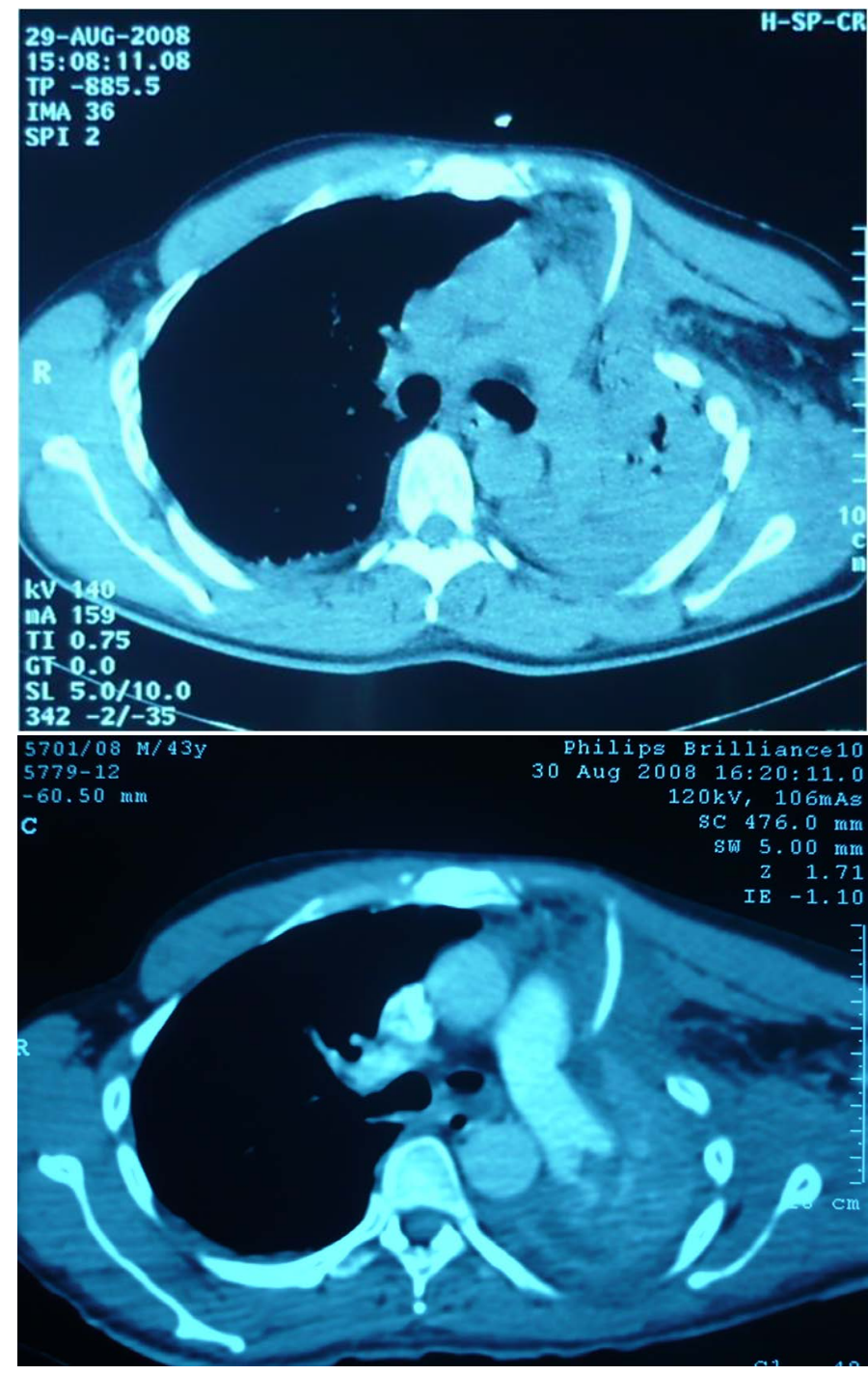

Figure 2 and 3: Thoracic CT scan without and with contrast injection. Intrathoracic curved rib coming into contact with left pulmonary artery, pulmonary contusion and pleural effusion.

Correspondence to: El Hassane Kabiri, Department of Thoracic Surgery, Mohamed V Military University Hospital, Riad 10100, Rabat, Morocco, Tel: 00 (212) 6 61262637; E-mail: hassankabiri@yahoo.com

Received: May 31, 2017; Accepted: June 22, 2017; Published: June 24, 2017 
After 3 weeks of the reanimation sojourn, the patient underwent a left thoracotomy through the $5^{\text {th }}$ intercostal space to remove endothoracic bone fragment which was actually in contact with the pulmonary artery causing an adventitial thickening. The postoperative evolution was simple.

\section{Discussion}

The present case involved a fracture of the anterior aspect of the rib with anterior displacement of the fragment and the proximity of the fracture to the groove for the pulmonary artery. These circumstances may have produced a pseudoaneurysm that caused delayed rupture of the artery. Angiography after the return of spontaneous circulation did not reveal any obvious vascular abnormalities.

Another mechanism by which delayed rupture of a pulmonary artery occurs is when the artery is directly injured by the jagged edge of a fractured bone. In the present case, body movement may have caused a jagged bone fragment to injure the nearby left pulmonary artery $[1,2]$.

Some intriguing cases of late-onset aortic injury after traumatic rib fractures have been reported [1-4]. The patients in all the cases developed vascular injury a few days after the onset of trauma and led to the diagnosis of direct aortic puncture by a fractured bone, which was supported by the operative findings. Similarly, from a case of acute onset fatal subclavian artery rupture associated with isolated clavicle fracture due to accidental injury while balding [5].

Physicians should bear in mind that bone fragments can result in vascular injury and even if most rib fractures can be managed conservatively, we should suggest rib fixation surgery or the resection of the rib edges, especially when the shape of the fractured bone seems at a high risk of vascular injury [2-6].

\section{References}

1. Kigawa I, Fukuda I, Fujii Y, Yamabuki K (1992) A sharp edge of the fractured ribs caused the aortic injury at bodyposition change: a case report. Nihon Kyobu Geka Gakkai Zasshi 40: 1116-1120. [Crossref]

2. Bruno VD, Batchelor TJ (2009) Late aortic injury: a rare complication of a posterior rib fracture. Ann Thorac Surg 87: 301-303. [Crossref]

3. Iyoda A, Satoh N, Yamakawa H, Fujino M, Hiroshima K, et al. (2003) Rupture of the descending thoracic aorta caused by blunt chest trauma: report of a case. Surg Today 33: 755-757. [Crossref]

4. Morimoto Y, Sugimoto T, Sakahira H, Matsuoka H, Yoshioka Y, et al. (2014) Successful management of threatened aortic rupture late after rib fracture caused by blunt chest trauma. Ann Vasc Surg 28: e11-13. [Crossref]

5. Kendall KM, Burton JH, Cushing B (2000) Fatal subclavian artery transection from isolated clavicle fracture. J Trauma 48: 316-318. [Crossref]

6. Wada T, Yasunaga H, Inokuchi R, Matsui H, Matsubara T, et al. (2015) Effectiveness of surgical rib fixation on prolonged mechanical ventilation in patients with traumatic rib fractures : a propensity score $\square$ matched analysis. J Crit Care 30: 1227-1231. [Crossref]

Copyright: (C2017 Hammoumi MEL. This is an open-access article distributed under the terms of the Creative Commons Attribution License, which permits unrestricted use, distribution, and reproduction in any medium, provided the original author and source are credited. 